\title{
An Effective Extension of the Wagner Hierarchy to Blind Counter Automata
}

\author{
Olivier Finkel \\ Equipe de Logique Mathématique \\ U.F.R. de Mathématiques, Université Paris 7 \\ 2 Place Jussieu 75251 Paris cedex 05, France \\ finkel@logique.jussieu.fr.
}

\begin{abstract}
The extension of the Wagner hierarchy to blind counter automata accepting infinite words with a Muller acceptance condition is effective. We determine precisely this hierarchy.
\end{abstract}

Keywords: $\omega$-languages; blind counter automata; effective extension of the Wagner hierarchy; topological properties; Wadge hierarchy; Wadge games.

\section{Introduction}

Regular $\omega$-languages are accepted by (deterministic) Muller automata. Finite machines having a stronger expressive power when reading infinite words have also been investigated [Sta97a]. Recently Engelfriet and Hoogeboom studied Xautomata, i.e. automata equipped with a storage type $\mathbf{X}$, including the cases of pushdown automata, Turing machines, Petri nets EH93. A way to investigate the expressive power of such machines is to study the topological complexity of the $\omega$-languages they accept. For deterministic machines, it is shown in EH93] that every $\mathbf{X}$-automaton accepts boolean combinations of $\boldsymbol{\Pi}_{2}^{\mathbf{0}}$-sets. Hence in order to distinguish the different storage types it turned out that the study of the Wadge hierarchy is suitable. The Wadge hierarchy is a great refinement of the Borel hierarchy, recently studied by Duparc Dup99a. The Wadge hierarchy of $\omega$-regular languages has been determined in an efective way by Wagner Wag79. Several extensions of this hierarchy have been recently determined as the extension to deterministic pushdown automata, to $k$-blind counter automata, DFR01 Dup99b Fin00b. We present here the extension to (one) blind counter automata, which is the first known effective extension. We study Muller blind counter automata (MBCA), and define chains and superchains as Wagner did for Muller automata. The essential difference between the two hierarchies relies on the existence of superchains of transfinite length $\alpha<\omega^{2}$ for MBCA. The hierarchy is effective and leads to effective winning strategies in Wadge games between MBCA. The hierarchy of Muller automata equipped with several blind counters is presented in a non effective way in Fin00b DFR01. 


\section{Regular and Blind Counter $\omega$-languages}

We assume the reader to be familiar with the theory of formal languages and of $\omega$-regular languages, see for example [HU69], Tho90]. We first recall some definitions and results concerning $\omega$-regular languages and omega pushdown automata and introduce blind counter automata as a special case of pushdown automata Tho90 Sta97a].

When $\Sigma$ is a finite alphabet, a finite string (word) over $\Sigma$ is any sequence $x=x_{1} \ldots x_{k}$, where $x_{i} \in \Sigma$ for $i=1, \ldots, k$, and $k$ is an integer $\geq 1$. The length of $x$ is $k$, denoted by $|x|$. If $|x|=0, x$ is the empty word denoted by $\lambda$.

we write $x(i)=x_{i}$ and $x[i]=x(1) \ldots x(i)$ for $i \leq k$ and $x[0]=\lambda . \Sigma^{\star}$ is the set of finite words over $\Sigma$. The first infinite ordinal is $\omega$. An $\omega$-word over $\Sigma$ is an $\omega$-sequence $a_{1} \ldots a_{n} \ldots$, where $a_{i} \in \Sigma, \forall i \geq 1$. When $\sigma$ is an $\omega$-word over $\Sigma$, we write $\sigma=\sigma(1) \sigma(2) \ldots \sigma(n) \ldots$ and $\sigma[n]=\sigma(1) \sigma(2) \ldots \sigma(n)$ the finite word of length n, prefix of $\sigma$. The set of $\omega$-words over the alphabet $\Sigma$ is denoted by $\Sigma^{\omega}$. An $\omega$-language over an alphabet $\Sigma$ is a subset of $\Sigma^{\omega}$.

The usual concatenation product of two finite words $u$ and $v$ is denoted $u . v$ (and sometimes just $u v$ ). This product is extended to the product $u . v$ of a finite word $u$ and an $\omega$-word $v$.

For $V \subseteq \Sigma^{\star}, V^{\omega}=\left\{\sigma=u_{1} \ldots u_{n} \ldots \in \Sigma^{\omega} / u_{i} \in V, \forall i \geq 1\right\}$ is the $\omega$-power of $V$.

R. Mc Naughton established that the expressive power of deterministic Muller automata (DMA) is equal to the expressive power of non deterministic Muller automata (MA) [Tho90. An $\omega$-language is regular iff it is accepted by a Muller automaton. The class $R E G_{\omega}$ of $\omega$-regular languages is the $\omega$-Kleene closure of the class $R E G$ of (finitary) regular languages where the $\omega$-Kleene closure of a family $\mathrm{L}$ of finitary languages is :

$$
\omega-K C(L)=\left\{\cup_{i=1}^{n} U_{i} \cdot V_{i}^{\omega} / U_{i}, V_{i} \in L, \forall i \in[1, n]\right\}
$$

We now define the (blind) one counter machines which we assume here to be realtime and deterministic, and the corresponding classes of blind counter $\omega$ languages.

Definition 1. A (realtime deterministic) pushdown machine (PDM) is a 6-tuple $M=\left(K, \Sigma, \Gamma, \delta, q_{0}, Z_{0}\right)$, where $K$ is a finite set of states, $\Sigma$ is a finite input alphabet, $\Gamma$ is the finite pushdown alphabet, $q_{0} \in K$ is the initial state, $Z_{0} \in \Gamma$ is the start symbol, and $\delta$ is a mapping from $K \times \Sigma \times \Gamma$ into $K \times \Gamma^{\star}$.

If $\gamma \in \Gamma^{+}$describes the pushdown store content, the leftmost symbol will be assumed to be on " top" of the store. A configuration of a PDM is a pair $(q, \gamma)$ where $q \in K$ and $\gamma \in \Gamma^{\star}$.

For $a \in \Sigma, \gamma, \beta \in \Gamma^{\star}$ and $Z \in \Gamma$, if $(p, \beta)$ is in $\delta(q, a, Z)$, then we write $a:(q, Z \gamma) \mapsto_{M}(p, \beta \gamma)$.

$\mapsto_{M}^{\star}$ is the transitive and reflexive closure of $\mapsto_{M}$. (The subscript $M$ will be 
omitted whenever the meaning remains clear).

Let $\sigma=a_{1} a_{2} \ldots a_{n} \ldots$ be an $\omega$-word over $\Sigma$. An infinite sequence of configurations $r=\left(q_{i}, \gamma_{i}\right)_{i \geq 1}$ is called a run of $M$ on $\sigma$, starting in configuration $(p, \gamma)$, iff:

1. $\left(q_{1}, \gamma_{1}\right)=(p, \gamma)$

2. for each $i \geq 1, a_{i}:\left(q_{i}, \gamma_{i}\right) \mapsto_{M}\left(q_{i+1}, \gamma_{i+1}\right)$

For every such run, In $(r)$ is the set of all states entered infinitely often during run $r$.

A run $r$ of $M$ on $\sigma$, starting in configuration $\left(q_{0}, Z_{0}\right)$, will be simply called " a run of $M$ on $\sigma$ ".

$A$ one counter machine is a PDM such that $\Gamma=\left\{Z_{0}, I\right\}$ where $Z_{0}$ is the bottom symbol and always remains at the bottom of the store. So the pushdown store is used like a counter whose value is the integer $n$ if the content of the pushdown store is $I^{n} Z_{0}$.

A one blind counter machine is a one counter machine such that every transition which is enabled at zero level is also enabled at non zero level, i.e. if $\delta\left(q, a, Z_{0}\right)=$ $\left(p, I^{n} Z_{0}\right)$, for some $p, q \in K, a \in \Sigma$ and $n \geq 0$, then $\delta(q, a, I)=\left(p, I^{n+1}\right)$. But the converse may not be true, i.e. some transition may be enabled at non zero level but not at zero level.

Definition 2. A Muller (realtime deterministic) blind counter automaton (MBCA) is a 7-tuple $\mathcal{A}=\left(K, \Sigma, \Gamma, \delta, q_{0}, Z_{0}, \mathcal{F}\right)$ where $\mathcal{A}^{\prime}=\left(K, \Sigma, \Gamma, \delta, q_{0}, Z_{0}\right)$ is a (realtime deterministic ) one blind counter machine and $\mathcal{F} \subseteq 2^{K}$ is the collection of designated state sets.

The $\omega$-language accepted by $M$ is $L(\mathcal{A})=\left\{\sigma \in \Sigma^{\omega} /\right.$ there exists a run $r$ of $\mathcal{A}$ on $\sigma$ such that $\operatorname{In}(r) \in \mathcal{F}\}$.

The class of $\omega$-languages accepted by $\mathbf{M B C A}$ will be denoted $\mathbf{B C}$.

Remark 3. Machines we call here one blind counter machines are sometimes called one partially blind counter machines as in [Gre78].

Remark 4. If $M$ is a deterministic pushdown machine, then for every $\sigma \in \Sigma^{\omega}$, there exists at most one run $r$ of $M$ on $\sigma$ determined by the starting configuration. Each $\omega$-language accepted by a Muller deterministic pushdown automaton (DMPDA) can be accepted by a DMPDA such that for every $\sigma \in \Sigma^{\omega}$, there exists such a run of $M$ on $\sigma$.

But this is not true for MBCA because some words $x$ may be rejected by an $M B C A \mathcal{A}$ because the machine $\mathcal{A}$ blocks at zero level when reading $x$. This is connected with the fact that the class $\mathbf{B C}$ is not closed under complementation as it is shown by the following example.

Example 5. It is easy to see that the $\omega$-language $L=\left\{a^{n} b^{p} c^{\omega} / p \leq n\right\}$ is accepted by a deterministic $M B C A$, but its complement is not accepted by any deterministic $M B C A$ because $L^{\prime}=\left\{a^{n} b^{p} c^{\omega} / p>n\right\}$ is not accepted by any deterministic $M B C A$. 


\section{Topology}

We assume the reader to be familiar with basic notions of topology which may be found in Kur66] LT94] Sta97a] PP98].

Topology is an important tool for the study of $\omega$-languages, and leads to characterization of several classes of $\omega$-languages.

For a finite alphabet $X$, we consider $X^{\omega}$ as a topological space with the Cantor topology (see [LT94 Sta97a [PP98). The open sets of $X^{\omega}$ are the sets in the form $W . X^{\omega}$, where $W \subseteq X^{\star}$. A set $L \subseteq X^{\omega}$ is a closed set iff its complement $X^{\omega}-L$ is an open set. The class of open sets of $X^{\omega}$ will be denoted by $\mathbf{G}$ or by $\Sigma_{1}^{0}$. The class of closed sets will be denoted by $\mathbf{F}$ or by $\boldsymbol{\Pi}_{1}^{0}$. Closed sets are characterized by the following:

Proposition 6. A set $L \subseteq X^{\omega}$ is a closed set of $X^{\omega}$ iff for every $\sigma \in X^{\omega}$, $\left[\forall n \geq 1, \exists u \in X^{\omega}\right.$ such that $\left.\sigma(1) \ldots \sigma(n) . u \in L\right]$ implies that $\sigma \in L$.

Define now the next classes of the Hierarchy of Borel sets of finite rank:

Definition 7. The classes $\boldsymbol{\Sigma}_{\mathbf{n}}^{\mathbf{0}}$ and $\boldsymbol{\Pi}_{\mathbf{n}}^{\mathbf{0}}$ of the Borel Hierarchy on the topological space $X^{\omega}$ are defined as follows:

$\boldsymbol{\Sigma}_{1}^{0}$ is the class of open sets of $X^{\omega}$.

$\Pi_{1}^{0}$ is the class of closed sets of $X^{\omega}$.

$\mathbf{\Pi}_{2}^{0}$ or $\mathbf{G}_{\delta}$ is the class of countable intersections of open sets of $X^{\omega}$.

$\boldsymbol{\Sigma}_{\mathbf{2}}^{\mathbf{0}}$ or $\mathbf{F}_{\sigma}$ is the class of countable unions of closed sets of $X^{\omega}$.

And for any integer $n \geq 1$ :

$\boldsymbol{\Sigma}_{\mathbf{n}+\mathbf{1}}^{\mathbf{1}}$ is the class of countable unions of $\boldsymbol{\Pi}_{\mathbf{n}}^{\mathbf{0}}$-subsets of $X^{\omega}$.

$\boldsymbol{\Pi}_{\mathbf{n}+\mathbf{1}}^{\mathbf{0}}$ is the class of countable intersections of $\boldsymbol{\Sigma}_{\mathbf{n}}^{\mathbf{0}}$-subsets of $X^{\omega}$.

There is a nice characterization of $\boldsymbol{\Pi}_{2}^{0}$-subsets of $X^{\omega}$. First define the notion of $W^{\delta}$ :

Definition 8. For $W \subseteq X^{\star}$, let:

$W^{\delta}=\left\{\sigma \in X^{\omega} / \exists^{\omega} i\right.$ such that $\left.\sigma[i] \in W\right\}$.

$\left(\sigma \in W^{\delta}\right.$ iff $\sigma$ has infinitely many prefixes in $\left.W\right)$.

Then we can state the following Proposition:

Proposition 9. A subset $L$ of $X^{\omega}$ is a $\boldsymbol{\Pi}_{2}^{0}$-subset of $X^{\omega}$ iff there exists a set $W \subseteq X^{\star}$ such that $L=W^{\delta}$.

Mc Naughton's Theorem implies that every $\omega$-regular language is a boolean combination of $G_{\delta}$-sets, hence a $\boldsymbol{\Delta}_{\mathbf{3}}^{\mathbf{0}}=\left(\boldsymbol{\Pi}_{\mathbf{3}}^{\mathbf{0}} \cap \boldsymbol{\Sigma}_{\mathbf{3}}^{\mathbf{0}}\right)$-set. This result holds in fact for every $\omega$-language accepted by a deterministic $\mathbf{X}$-automaton in the sense of EH93, i.e. an automaton equipped with a storage type $\mathbf{X}$, including the case of the Turing machine. A way to distinguish the expressive power of finite machines reading $\omega$-words is the Wadge hierarchy which we now introduce. 
Definition 10. For $E \subseteq X^{\omega}$ and $F \subseteq Y^{\omega}, E$ is said to be Wadge reducible to $F\left(E \leq_{W} F\right)$ iff there exists a continuous function $f: X^{\omega} \rightarrow Y^{\omega}$, such that $E=f^{-1}(F)$.

$E$ and $F$ are Wadge equivalent iff $E \leq_{W} F$ and $F \leq_{W} E$. This will be denoted by $E \equiv_{W} F$. And we shall say that $E<_{W} F$ iff $E \leq_{W} F$ but not $F \leq_{W} E$.

$A$ set $E \subseteq X^{\omega}$ is said to be self dual iff $E \equiv_{W}\left(X^{\omega}-E\right)$, and otherwise it is said to be non self dual.

The relation $\leq_{W}$ is reflexive and transitive, and $\equiv_{W}$ is an equivalence relation. The equivalence classes of $\equiv_{W}$ are called wadge degrees.

$W H$ is the class of Borel subsets of finite rank of a set $X^{\omega}$, where $X$ is a finite set, equipped with $\leq_{W}$ and with $\equiv_{W}$.

For $E \subseteq X^{\omega}$ and $F \subseteq Y^{\omega}$, if $E \leq_{W} F$ and $E=f^{-1}(F)$ where $f$ is a continuous function from $X^{\omega}$ into $Y^{\omega}$, then $f$ is called a continuous reduction of $E$ to $F$. Intuitively it means that $E$ is less complicated than $F$ because to check whether $x \in E$ it suffices to check whether $f(x) \in F$ where $f$ is a continuous function. Hence the Wadge degree of an $\omega$-language is a measure of its topological complexity.

Remark 11. In the above definition, we consider that a subset $E \subseteq X^{\omega}$ is given together with the alphabet $X$. This is necessary as it is shown by the following example.

Let $E=\{0,1\}^{\omega}$ considered as an $\omega$-language over the alphabet $X=\{0,1\}$ and let $F=\{0,1\}^{\omega}$ be the same $\omega$-language considered as an $\omega$-language over the alphabet $Y=\{0,1,2\}$. Then $E$ is an open and closed subset of $\{0,1\}^{\omega}$ but $F$ is a closed and non open subset of $\{0,1,2\}^{\omega}$. It is easy to check that $E<_{W} F$ hence $E$ and $F$ are not Wadge equivalent.

Then we can define the Wadge class of a set $F$ :

Definition 12. Let $F$ be a subset of $X^{\omega}$. The wadge class of $F$ is $[F]$ defined by: $[F]=\left\{E / E \subseteq Y^{\omega}\right.$ for a finite alphabet $Y$ and $\left.E \leq_{W} F\right\}$.

Recall that each Borel class $\boldsymbol{\Sigma}_{\mathbf{n}}^{\mathbf{0}}$ and $\boldsymbol{\Pi}_{\mathbf{n}}^{\mathbf{0}}$ is a Wadge class.

There is a close relationship between Wadge reducibility and games which we now introduce. Define first the Wadge game $W(A, B)$ for $A \subseteq X_{A}^{\omega}$ and $B \subseteq X_{B}^{\omega}$ :

Definition 13. The Wadge game $W(A, B)$ is a game with perfect information between two players, player 1 who is in charge of $A$ and player 2 who is in charge of $B$.

Player 1 first writes a letter $a_{1} \in X_{A}$, then player 2 writes a letter $b_{1} \in X_{B}$, then player 1 writes a letter $a_{2} \in X_{A}$, and so on ...

The two players alternatively write letters $a_{n}$ of $X_{A}$ for player 1 and $b_{n}$ of $X_{B}$ for player 2.

After $\omega$ steps, the player 1 has written an $\omega$-word $a \in X_{A}^{\omega}$ and the player 2 has written an $\omega$-word $b \in X_{B}^{\omega}$.

The player 2 is allowed to skip, even infinitely often, provided he really write an 
$\omega$-word in $\omega$ steps.

The player 2 wins the play iff $[a \in A \leftrightarrow b \in B]$, i.e. iff

[ $(a \in A$ and $b \in B)$ or $(a \notin A$ and $b \notin B$ and $b$ is infinite $)]$.

Recall that a strategy for player 1 is a function $\sigma:\left(X_{B} \cup\{s\}\right)^{\star} \rightarrow X_{A}$. And a strategy for player 2 is a function $f: X_{A}^{+} \rightarrow X_{B} \cup\{s\}$.

$\sigma$ is a winning stategy (w.s.) for player 1 iff he always wins a play when he uses the strategy $\sigma$, i.e. when the $n^{t h}$ letter he writes is given by $a_{n}=\sigma\left(b_{1} \ldots b_{n-1}\right)$, where $b_{i}$ is the letter written by player 2 at step $i$ and $b_{i}=s$ if player 2 skips at step $i$.

A winning strategy for player 2 is defined in a similar manner.

Martin's Theorem states that every Gale-Stewart Game $G(X)$ (see [Tho90] PP98 for more details), with $X$ a borel set, is determined and this implies the following :

Theorem 14 (Wadge). Let $A \subseteq X_{A}^{\omega}$ and $B \subseteq X_{B}^{\omega}$ be two Borel sets, where $X_{A}$ and $X_{B}$ are finite alphabets. Then the Wadge game $W(A, B)$ is determined: one of the two players has a winning strategy. And $A \leq_{W} B$ iff the player 2 has a winning strategy in the game $W(A, B)$.

Recall that a set $X$ is well ordered by a binary relation $<$ iff $<$ is a linear order on $X$ and there is not any strictly decreasing $($ for $<$ ) infinite sequence of elements in $X$.

Theorem 15 (Wadge). Up to the complement and $\equiv_{W}$, the class of Borel subsets of finite rank of $X^{\omega}$, for $X$ a finite alphabet, is a well ordered hierarchy. There is an ordinal $|W H|$, called the length of the hierarchy, and a map $d_{W}^{0}$ from $W H$ onto $|W H|$, such that for all $A, B \in W H$ :

$d_{W}^{0} A<d_{W}^{0} B \leftrightarrow A<_{W} B$ and

$d_{W}^{0} A=d_{W}^{0} B \leftrightarrow\left[A \equiv_{W} B\right.$ or $\left.A \equiv_{W} B^{-}\right]$.

Remark 16. We do not give here the ordinal $|W H|$. Details may be found in Dup99a.

\section{Wagner Hierarchy and its Extension to Blind Counter Automata}

Consider now $\omega$-regular languages. Landweber studied first the topological properties of $\omega$-regular languages. He characterized the $\omega$-regular languages in each of the Borel classes $\mathbf{F}, \mathbf{G}, \mathbf{F}_{\sigma}, \mathbf{G}_{\delta}$, and showed that one can decide, for an effectively given $\omega$-regular language $L$, whether $L$ is in $\mathbf{F}, \mathbf{G}, \mathbf{F}_{\sigma}$, or $\mathbf{G}_{\delta}$.

It turned out that an $\omega$-regular language is in the class $\mathbf{G}_{\delta}$ iff it is accepted by a deterministic Büchi automaton. These results were refined by K. Wagner who studied the Wadge Hierarchy of $\omega$-regular languages. In fact there is an effective version of the Wadge Hierarchy restricted to $\omega$-regular languages: 
Theorem 17 (Corollary of Büchi-Landweber's Theorem [BL69]). For $A$ and $B$ some $\omega$-regular sets, one can effectively decide which player has a w.s. in the game $W(A, B)$ and the winner has a w.s. given by a transducer.

The hierarchy obtained on $\omega$-regular languages is now called the Wagner hierarchy and has length $\omega^{\omega}$. Wagner Wag79 gave an automata structure characterization, based on notion of chain and superchain, for an automaton to be in a given class and showed that the Wadge degree of an $\omega$-regular language is computable. Wilke and Yoo proved in WY95] that this can be done in polynomial time. Wagner's hierarchy has been recently studied by Carton and Perrin in connection with the theory of $\omega$-semigroups [CP97 CP98 [PP98 and by Selivanov in $[$ Sel98.

We present in this paper an extension of the Wagner hierarchy to the class of blind counter $\omega$-languages, using analogous notions of chains and superchains. We shall first define positive and negative loops, next chains and superchains. A crucial fact which allows this definition is the following lemma:

Lemma 18. Let $\mathcal{A}=\left(K, \Sigma, \Gamma, \delta, q_{0}, Z_{0}, \mathcal{F}\right)$ be a $M B C A$ and $x \in \Sigma^{\omega}$ such that there exists an infinite run $r=\left(q_{i}, I^{n_{i}} Z_{0}\right)_{i \geq 1}$ of $\mathcal{A}$ over $x$ such that $\operatorname{Inf}(r)=$ $F \subseteq K$. Then there exist infinitely many integers $i$ such that for all $j \geq i$, $n_{j} \geq n_{i}$. Among these integers there exist infinitely many integers $i_{k}, k \geq 1$, and a state $q \in K$ such that for all $k \geq 1, q_{i_{k}}=q$. Then there exist two integers $s, s^{\prime}$ such that between steps $i_{s}$ and $i_{s^{\prime}}$ of the run $r, \mathcal{A}$ enters in every state of $F$ and in not any other state of $K$, because $\operatorname{Inf}(r)=F$.

Proof. With the hypotheses of the lemma, assume that $r=\left(q_{i}, I^{n_{i}} Z_{0}\right)_{i>1}$ is an infinite run of $M$ over $x$. If there exist only finitely many integers $i$ such that for all $j \geq i, n_{j} \geq n_{i}$, then there exists a largest one $l$. But then if $j_{0}$ is an integer $>l$ there exists an integer $j_{1}>j_{0}$ such that $n_{j_{1}}<n_{j_{0}}$. By induction one could construct a sequence of integers $\left(j_{k}\right)_{k \geq 0}$ such that for all $k, n_{j_{k+1}}<n_{j_{k}}$. This would lead to a contradiction because every integer $n_{i}$ is positive.

Then there exist infinitely many integers $i$ such that $\forall j \geq i, n_{j} \geq n_{i}$. The set of states is finite, hence there exists a state $q \in K$ and infinitely many such integers $i_{k}, k \geq 1$, such that for all $k \geq 1, q_{i_{k}}=q$ and $n_{i_{k}}>0$ or for all $k \geq 1, q_{i_{k}}=q$ and $n_{i_{k}}=0$. Now if $\operatorname{Inf}(r)=F$, the states not in $F$ occur only finitely many times during run $r$ thus there exist two integers $s<s^{\prime}$ such that the set of states $\mathcal{A}$ enters between steps $i_{s}$ and $i_{s^{\prime}}$ of the run $r$ is exactly $F$.

Remark 19. The proof of Lemma 18 relies on a simple property of local minima of functions mapping natural numbers to themselves. A similar argument is due to Linna Lin77].

Then we shall write

(a) $(q, I) \stackrel{F}{\mapsto}^{\star}\left(q, I^{+}\right) \quad$ if $n_{i_{s}}>0$ and $n_{i_{s^{\prime}}}>n_{i_{s}}$

(b) $(q, I) \stackrel{F}{\mapsto}^{\star}\left(q, I^{=}\right) \quad$ if $n_{i_{s}}>0$ and $n_{i_{s^{\prime}}}=n_{i_{s}}$ 
(c) $\left(q, Z_{0}\right) \stackrel{F}{\mapsto}^{\star}\left(q, Z_{0}\right) \quad$ if $n_{i_{s}}=0$ and $n_{i_{s^{\prime}}}=0$

The set $F$ is said to be an essential set (of states) and we shall say that in the case (a) there exists a loop $L(q, I, F,+)$, in the case (b) there exists a loop $L(q, I, F,=$ ), in the case $(c)$ there exists a loop $L\left(q, Z_{0}, F,=\right)$. Such a loop is positive if $F \in \mathcal{F}$ and it is negative if $F \notin \mathcal{F}$. We then denote the loop $L(q, I, F,=)$ by $L^{+}(q, I, F,=)$ or $L^{-}(q, I, F,=)$ and similarly in the other cases.

Lemma 20. The set of essential sets and the set of positive and negative loops of a MBCA is effectively computable.

This follows from the decidability of the emptiness problem for context free languages accepted by pushdown automata.

We assume now some familiarity with the Wagner hierarchy as presented in Wag79 Sta97a. The next step is to define, following Wagner's study, the (alternating) chains. Let $E^{+}$(respectively $E^{-}$) be the set of essential sets in $\mathcal{F}$ (respectively not in $\mathcal{F}$ ). An alternating chain of length $n$ is in the form

$$
F_{1} \subset F_{2} \subset F_{3} \subset \ldots F_{n}
$$

where $F_{i} \in E^{+}$iff $F_{i+1} \in E^{-}$for $1 \leq i<n$. It is a positive chain if $F_{1} \in E^{+}$ and a negative chain if $F_{1} \in E^{-}$.

As in the case of Muller automata Sta97a, one can see that if $F$ is a maximal essential set then all (alternating) chains of maximal length contained in $F$ have the same sign (positive or negative) because in every chain of maximal length contained in $F$ one can replace the last essential set by $F$ itself. Let then $l(F)$ be the maximal length of chains contained in $F$ and $s(F)$ be the sign of these chains.

We now define the first invariant of the MBCA $\mathcal{A}$ as $\mathrm{m}(\mathcal{A})$ being the maximal length of chains of essential sets. Lemma 18 is crucial because it makes every essential set $F_{i}$ of a chain $F_{1} \subset F_{2} \subset F_{3} \subset \ldots F_{n}$ to be indefinitely reachable from $(q, I)$ ( respectively $\left.\left(q, Z_{0}\right)\right)$ if there exists a loop $L\left(q, I, F_{n},+\right.$ or $\left.=\right)$, ( respectively $\left.L\left(q, Z_{0}, F_{n},=\right)\right)$.

The great difference between the case of Muller automata and the case of MBCA comes with the notion of superchain. Briefly speaking in a MA $\mathcal{A}$ a superchain of length $n$ is a sequence $S_{1}, \ldots, S_{n}$ of chains of length $\mathrm{m}(\mathcal{A})$ such that for every integer $i, 1 \leq i<n, S_{i+1}$ is reachable from $S_{i}$ and $S_{i+1}$ is positive iff $S_{i}$ is negative. In the case of MA, $S_{i}$ cannot be reachable from $S_{i+1}$ otherwise there would exist a chain of length $>\mathrm{m}(\mathcal{A})$.

But in the case of MBCA, in such a superchain, $S_{i}$ may be reachable from $S_{i+1}$ but with a reachability which is limited by the counter. This leads to the notion of superchains of length $\omega$, where $\omega$ is the first infinite ordinal, and next of length $\alpha$ where $\alpha$ is an ordinal $<\omega^{2}$. 
An example of a MBCA $\mathcal{A}$ with $\mathrm{m}(\mathcal{A})=m$ and a superchain of length $\omega$ is obtained from two MA $\mathcal{B}$ and $\mathcal{B}^{\prime}$ such that the graph of $\mathcal{B}$ is just constituted by a positive chain of length $m$ with a maximal essential set $F_{m}=\left\{q_{1}, \ldots q_{m}\right\}$ and the graph of $\mathcal{B}^{\prime}$ is just constituted by a negative chain of length $m$ with a maximal essential set $F_{m}^{\prime}=\left\{q_{1}^{\prime}, \ldots q_{m}^{\prime}\right\}$. The behaviour of the MBCA $\mathcal{A}$ is as follows: at the beginning of an infinite run, the counter may be increased up to a counter value $N$; then there exist transitions from state $q_{1}$ to $q_{1}^{\prime}$ and conversely from state $q_{1}^{\prime}$ to $q_{1}$ but these transitions make the counter value decrease. Moreover $\mathcal{A}$ has also the transitions of the two MA $\mathcal{B}$ and $\mathcal{B}^{\prime}$ but these transitions do not change the counter value. Then one can see thet after a first transition from state $q_{1}$ to $q_{1}^{\prime}$ or from $q_{1}^{\prime}$ to $q_{1}$ the number of such transitions is bounded by the counter value $N$, but this initial value may be chosen $>n_{0}$ where $n_{0}$ is any given integer.

Let then $\mathcal{A}$ be a MBCA such that $\mathrm{m}(\mathcal{A})=m$ and such that $\mathcal{A}$ has positive and negative chains of length $m$. A superchain of length $\omega$ is formed by two maximal loops $L^{+}\left(q, I, F_{m},+o r=\right)$ and $L^{-}\left(q^{\prime}, I, F_{m}^{\prime},+o r=\right)$ of such chains, i.e. $F_{m}$ is the last element of a positive chain of length $m$ and $F_{m}^{\prime}$ is the last element of a negative chain of length $m$; moreover, for all $p_{0}>1$, configurations $\left(q, I^{p} Z_{0}\right)$ are reachable for integers $p>p_{0}$, and there exist transitions implying that

$$
\left(q, I^{p} Z_{0}\right) \mapsto^{\star}\left(q^{\prime}, I^{p^{\prime}} Z_{0}\right) \mapsto^{\star}\left(q, I^{p^{\prime \prime}} Z_{0}\right)
$$

for some integers $p, p^{\prime}, p^{\prime \prime}$. the MBCA $\mathcal{A}$ having not any chain of length $>m$, it holds that $p^{\prime \prime}<p$, because otherwise there would exist an essential set $F \supseteq F_{m} \cup F_{m}^{\prime}$ and then there would exist a chain of length $>m$. And the loop $L^{+}\left(q, I, F_{m},+\right.$ or $\left.=\right)$ is in fact $L^{+}\left(q, I, F_{m},=\right)$ and similarly $L^{-}\left(q^{\prime}, I, F_{m}^{\prime},+o r=\right.$ ) is $L^{-}\left(q^{\prime}, I, F_{m}^{\prime},=\right)$

One can informally say that $F_{m}$ is reachable from $F_{m}^{\prime}$ and conversely but after such transitions the counter value has decreased hence there is a limitation to this reachability.

Lemma 21. The set of superchains of length $\omega$ of a MBCA is effectively computable.

Now one can define superchains of length $\omega \cdot p$ for an integer $p \geq 1$. Informally speaking a superchain of length $\omega \cdot p$ is a sequence $\Omega_{1}, \ldots, \Omega_{p}$ of superchains of length $\omega$ such that any state $q$ of an essential set of $\Omega_{i+1}$ is reachable with unbounded values of the counter from any state of an essential set of $\Omega_{i}$. It is now easy to define superchains of length $\omega \cdot p+s \geq 1$, (with $p, s$ some integers $\geq 0$ ), which are a sequence of a superchain of length $s$ followed by a superchain of length $\omega . p$.

In the case $s>0$, the superchain is said to be positive if it begins with a positive chain and it is said to be negative if it begins with a negative chain.

In the case $s=0$, we consider now that a superchain: $\Omega_{1}, \ldots, \Omega_{p}$, of length $\omega . p$, is given with a loop $L$. Then it is said to be positive (respectively, negative) if $\Omega_{1}$ 
is formed by two maximal loops $L^{+}\left(q, I, F_{m},=\right)$ and $L^{-}\left(q^{\prime}, I, F_{m}^{\prime},=\right)$ of chains of length $\mathrm{m}(\mathcal{A})=m$ and configurations $\left(q, I^{p} Z_{0}\right)$ are reachable for unbounded values of $p \geq 1$ from the positive loop $L$ (respectively, from the negative loop $L)$.

We define now the second invariant of the $\mathrm{MBCA} \mathcal{A}$ as $\mathrm{n}(\mathcal{A})$ being the maximal length of superchains $\left(\mathrm{n}(\mathcal{A})<\omega^{2}\right)$. The MBCA is said to be prime if all superchains of length $\mathrm{n}(\mathcal{A})$ have the same sign, i.e. all are positive or all are negative. Denote $\mathrm{s}(\mathcal{A})=0$ if $\mathcal{A}$ is not prime, $\mathrm{s}(\mathcal{A})=1$ if all longest superchains are positive, and $\mathrm{s}(\mathcal{A})=-1$ if all longest superchains are negative.

Lemma 22. Let $\mathcal{A}$ be a $M B C A$. Then $n(\mathcal{A})$ and $s(\mathcal{A})$ are computable. Moreover the set of superchains of length $n(\mathcal{A})$ is computable.

We can now follow Wagner's study and define for $\alpha$ an ordinal $<\omega^{2}$ and $m$ an integer $\geq 1$ :

$C_{m}^{\alpha}=\{L(\mathcal{A}) / \mathrm{s}(\mathcal{A})=1$ and $\mathrm{m}(\mathcal{A})=m$ and $\mathrm{n}(\mathcal{A})=\alpha\}$

$D_{m}^{\alpha}=\{L(\mathcal{A}) / \mathrm{s}(\mathcal{A})=-1$ and $\mathrm{m}(\mathcal{A})=m$ and $\mathrm{n}(\mathcal{A})=\alpha\}$

$E_{m}^{\alpha}=\{L(\mathcal{A}) / \mathrm{s}(\mathcal{A})=0$ and $\mathrm{m}(\mathcal{A})=m$ and $\mathrm{n}(\mathcal{A})=\alpha\}$

Using the Wadge game, one can now show that each class $C_{m}^{\alpha}$ or $D_{m}^{\alpha}$ defines a Wadge degree, i.e. all $\omega$-languages in the same class $C_{m}^{\alpha}$ or $D_{m}^{\alpha}$ are Wadge equivalent. In other words $C_{m}^{\alpha}$ and $D_{m}^{\alpha}$ are the restrictions to the class $\mathbf{B C}$ of some Wadge degrees.

Moreover when $\alpha=n$ is an integer, this degree corresponds to the degree obtained in the Wagner hierarchy for the classes $C_{m}^{n}$ or $D_{m}^{n}$.

The classes $C_{m}^{\alpha}, D_{m}^{\alpha}$, and $E_{m}^{\alpha}$, for $m$ an integer $\geq 1$ and $\alpha$ a non null ordinal $<\omega^{2}$, form the coarse structure of the Wadge hierarchy of BC. It is a strict extension of the coarse structure of the Wagner hierarchy studied in Wag79 and it satisfies the following Theorem.

Theorem 23. Let $\mathcal{A}$ and $\mathcal{B}$ be two $M B C A$ accepting the $\omega$-languages $L(\mathcal{A})$ and $L(\mathcal{B})$. Then it holds that:

1. If $m(\mathcal{A})<m(\mathcal{B})$, then $L(\mathcal{A})<_{W} L(\mathcal{B})$.

2. If $m(\mathcal{A})=m(\mathcal{B})$, and $n(\mathcal{A})<n(\mathcal{B})$, then $L(\mathcal{A})<_{W} L(\mathcal{B})$.

3. If $m(\mathcal{A})=m(\mathcal{B}), n(\mathcal{A})=n(\mathcal{B}), s(\mathcal{A})=1$ or $s(\mathcal{A})=-1$, and $s(\mathcal{B})=0$, then $L(\mathcal{A})<_{W} L(\mathcal{B})$.

4. If $m(\mathcal{A})=m(\mathcal{B}), n(\mathcal{A})=n(\mathcal{B}), s(\mathcal{A})=1$ and $s(\mathcal{B})=-1$, then $L(\mathcal{A})$ and $L(\mathcal{B})$ are non self dual and $L(\mathcal{A}) \equiv_{W} L(\mathcal{B})^{-}$.

From this Theorem one can easily infer that the integer $\mathrm{m}(\mathcal{A})$, the ordinal $\mathrm{n}(\mathcal{A})$, and $\mathrm{s}(\mathcal{A}) \in\{-1,0,1\}$, are invariants of the $\omega$-language $L(\mathcal{A})$ and not only of the MBCA $\mathcal{A}$ : 
Corollary 24. Let $\mathcal{A}$ and $\mathcal{B}$ be two $M B C A$ accepting the same $\omega$-language $L(\mathcal{A})=$ $L(\mathcal{B})$. Then $m(\mathcal{A})=m(\mathcal{B}), n(\mathcal{A})=n(\mathcal{B})$, and $s(\mathcal{A})=s(\mathcal{B})$.

One can give a canonical member in each of the classes $C_{m}^{\alpha}, D_{m}^{\alpha}$, and $E_{m}^{\alpha}$, for $m$ an integer $\geq 1$ and $\alpha$ a non null ordinal $<\omega^{2}$. And one can easily deduce that the length of the coarse structure of the Wadge hierarchy of blind counter $\omega$-languages is the ordinal $\omega^{3}$, while the length of the coarse structure of the Wagner hierarchy was the ordinal $\omega^{2}$.

The coarse structure of the class $\mathbf{B C}$ is effective but it is not exactly the Wadge hierarchy of $\mathbf{B C}$, because each class $E_{m}^{\alpha}$ is the union of countably many (restrictions of) Wadge degrees. We can next define a sort of derivation as Wagner did for Muller automata.

Two MBCA $\mathcal{A}$ and $\mathcal{B}$ in the same class $E_{m}^{\alpha}$ have essentially the same "most difficult parts" because they have positive and negative superchains of length $\mathrm{n}(\mathcal{A})=\mathrm{n}(\mathcal{B})$. Hence, in the case of Muller automata (then $\alpha$ is an integer), Wagner's idea was to cut off the superchains of length $\mathrm{n}(\mathcal{A})=\mathrm{n}(\mathcal{B})$ of $\mathcal{A}$ and $\mathcal{B}$; this way one get some new automata $\partial \mathcal{A}$ and $\partial \mathcal{B}$ which are called the derivations of $\mathcal{A}$ and $\mathcal{B}$ and the comparison of $\mathcal{A}$ and $\mathcal{B}$ with regard to $\leq_{W}$ is reduced to the comparison of their derivations $\partial \mathcal{A}$ and $\partial \mathcal{B}$.

In the case of MBCA one do as in the case of MA but with some modification. We first define the derivation $\partial \mathcal{A}$ of a MBCA in $E_{m}^{\alpha}: \mathcal{A}=\left(K, \Sigma, \Gamma, \delta, q_{0}, Z_{0}, \mathcal{F}\right)$ as follows.

Let $\partial K$ be the set of states in $K$ from which some positive and some negative superchains of length $\mathrm{n}(\mathcal{A})$ are reachable. In fact for each such $q \in \partial K$, it may exist an integer $n_{q}$ such that positive and negative superchains of length $\mathrm{n}(\mathcal{A})$ are reachable only from configurations $\left(q, I^{n} Z_{0}\right)$ with $n \geq n_{q}$. And these integers $n_{q}$ are effectively computable. Let us define now

$$
\partial \mathcal{A}=\left(\partial K, \Sigma, \Gamma=\left\{I, Z_{0}\right\}, \partial \delta, q_{0}, Z_{0}, \partial \mathcal{F}\right)
$$

where $\partial \delta$ is defined by:

for each $q \in \partial K, a \in \Sigma, Z \in \Gamma$ :

$\partial \delta(q, a, Z)=\delta(q, a, Z)$ if $\delta(q, a, Z)=(p, \gamma)$ for some $\gamma \in \Gamma^{\star}$ and $p \in \partial K$.

Otherwise $\partial \delta(q, a, Z)$ is undefined.

And $\partial \mathcal{F}=\{F / F \subseteq \partial K$ and $F \in \mathcal{F}\}$

We consider now the MBCA $\partial \mathcal{A}$ given with the integers $n_{q}$, for $q \in \partial K$. Then we study the loops of $\partial \mathcal{A}$ as above but we keep only loops in the form $L\left(q, Z_{0}\right.$ or $\left.I, F,+o r-\right)$ such that state $q$ is reachable with a counter value $n \geq n_{q}$. We can next define chains and superchains for $\partial^{\prime} \mathcal{A}=\left(\partial \mathcal{A},\left(n_{q}\right)_{q \in \partial K}\right)$. We define $\mathrm{m}\left(\partial^{\prime} \mathcal{A}\right), \mathrm{n}\left(\partial^{\prime} \mathcal{A}\right)$, and $\mathrm{s}\left(\partial^{\prime} \mathcal{A}\right)$, and it holds that $\mathrm{m}\left(\partial^{\prime} \mathcal{A}\right)<\mathrm{m}(\mathcal{A})$. We then 
attribute a class $C_{\mathrm{m}\left(\partial^{\prime} \mathcal{A}\right)}^{\mathrm{n}\left(\partial^{\prime} \mathcal{A}\right.}, D_{\mathrm{m}\left(\partial^{\prime} \mathcal{A}\right)}^{\mathrm{n}\left(\partial^{\prime} \mathcal{A}\right)}$, or $E_{\mathrm{m}\left(\partial^{\prime} \mathcal{A}\right)}^{\mathrm{n}\left(\partial^{\prime} \mathcal{A}\right)}$, to $\partial^{\prime} \mathcal{A}$ as we did for $\mathcal{A}$. It may happen that there does not exist any loop for $\partial^{\prime} \mathcal{A}=\left(\partial \mathcal{A},\left(n_{q}\right)_{q \in \partial K}\right)$; in that case we associate the class $E$ to $\partial^{\prime} \mathcal{A}$. Now we can iterate this process and associate to the MBCA $\mathcal{A}$ a name $N(\mathcal{A})$ which is inductively defined by:

1. If $\mathcal{A}$ is prime and $\mathrm{s}(\mathcal{A})=1$, then $N(\mathcal{A})=C_{\mathrm{m}(\mathcal{A})}^{\mathrm{n}(\mathcal{A})}$.

2. If $\mathcal{A}$ is prime and $\mathrm{s}(\mathcal{A})=-1$, then $N(\mathcal{A})=D_{\mathrm{m}(\mathcal{A})}^{\mathrm{n}(\mathcal{A})}$.

3. If $\mathcal{A}$ is not prime then $N(\mathcal{A})=E_{\mathrm{m}(\mathcal{A})}^{\mathrm{n}(\mathcal{A})} N\left(\partial^{\prime} \mathcal{A}\right)$.

This name depends only on the $\omega$-language $L(\mathcal{A})$ accepted by the MBCA $\mathcal{A}$ and is effectively computable. We can write it in a similar fashion as in Wagner's study: we associate with each blind counter $\omega$-language $\mathrm{L}(\mathcal{A})$ in $\mathbf{B C}$ a name in the form:

$$
N(\mathcal{A})=E_{m_{1}}^{\alpha_{1}} \ldots E_{m_{k}}^{\alpha_{k}} H_{m_{k+1}}^{\alpha_{k+1}}
$$

where $m_{1}>m_{2}>\ldots>m_{k}>m_{k+1}$ are integers; each $\alpha_{i}$ is an ordinal $<\omega^{2}$; and $H \in\{C, D\}$, or in the form:

$$
N(\mathcal{A})=E_{m_{1}}^{\alpha_{1}} \ldots E_{m_{k}}^{\alpha_{k}} E
$$

which we shall simply denote by

$$
N(\mathcal{A})=E_{m_{1}}^{\alpha_{1}} \ldots E_{m_{k}}^{\alpha_{k}}
$$

where $m_{1}>m_{2}>\ldots>m_{k}$ are integers and each $\alpha_{i}$ is an ordinal $<\omega^{2}$.

One can show that each such name is really the name of an $\omega$-language in $\mathbf{B C}$. And the Wadge relation $\leq_{W}$ is now computable because of the following result.

Theorem 25. Let $\mathcal{A}$ and $\mathcal{B}$ be two $M B C A$ accepting the $\omega$-languages $L(\mathcal{A})$ and $L(\mathcal{B})$. Assume that the names associated with the $M B C A \mathcal{A}$ and $\mathcal{B}$ are:

$$
\begin{gathered}
N(\mathcal{A})=E_{m_{1}}^{\alpha_{1}} \ldots E_{m_{k}}^{\alpha_{k}} H_{m_{k+1}}^{\alpha_{k+1}} \\
N(\mathcal{B})=E_{m_{1}^{\prime}}^{\alpha_{1}^{\prime}} \ldots E_{m_{l}^{\prime}}^{\alpha_{l}^{\prime}} H_{m_{l+1}^{\prime} \alpha_{l+1}^{\prime}}^{\alpha_{l}^{\prime}}
\end{gathered}
$$

where $(H=E$ or $H=C$ or $H=D)$, and $\left(H^{\prime}=E\right.$ or $H^{\prime}=C$ or $\left.H^{\prime}=D\right)$.

Then $L(\mathcal{A}) \leq_{W} L(\mathcal{B})$ if there exists an integer $j \leq \min (k+1, l+1)$ such that $m_{i}=m_{i}^{\prime}$ and $n_{i}=n_{i}^{\prime}$ for $1 \leq i \leq j$ and one of the two following properties holds.

1. $j=k+1 \leq l+1$ and $H^{\prime}=E$ or $H=H^{\prime}$.

2. $j<\min (k+1, l+1)$ and

$$
m_{j+1}<m_{j+1}^{\prime} \text { or }\left(m_{j+1}=m_{j+1}^{\prime} \text { and } \alpha_{j+1}<\alpha_{j+1}^{\prime}\right) \text {. }
$$


Then the structure of the Wadge hierarchy of $\omega$-languages in $\mathbf{B C}$ is completely determined. One can show that a blind counter $\omega$-language $L(\mathcal{A})$, where $\mathcal{A}$ is a MBCA, is in the class $\Delta_{2}^{0}$ iff $\mathrm{m}(\mathcal{A})<2$, i.e. iff the name of $\mathcal{A}$ is in the form $C_{1}^{\alpha}, D_{1}^{\alpha}$, or $E_{1}^{\alpha}$, for $\alpha<\omega^{2}$. Thus the Wadge hierarchy restricted to the class $\mathbf{B C} \cap \boldsymbol{\Delta}_{\mathbf{2}}^{\mathbf{0}}$ has length $\omega^{2}$, while the Wadge hierarchy restricted to $R E G_{\omega} \cap \boldsymbol{\Delta}_{\mathbf{2}}^{\mathbf{0}}$ has length $\omega$. The Wadge hierarchy of $\mathbf{B C} \cap \boldsymbol{\Delta}_{2}^{0}$ is then a great extension of the Wagner hierarchy restricted to the class $\boldsymbol{\Delta}_{2}^{0}$. This phenomenon is still true for larger Wadge degrees and non $\boldsymbol{\Delta}_{2}^{0}$-sets. Considering the length of the whole hierarchy of $\mathbf{B C}$ we get the following:

Corollary 26. (a) The length of the Wadge hierarchy of blind counter $\omega$ languages in $\boldsymbol{\Delta}_{\mathbf{2}}^{\mathbf{0}}$ is $\omega^{2}$.

(b) The length of the Wadge hierarchy of blind counter $\omega$-languages is the ordinal $\omega^{\omega}$ (hence it is equal to the length of the Wagner hierarchy).

Once the structures of two MBCA $\mathcal{A}$ and $\mathcal{B}$ are determined as well as their names $N(\mathcal{A})$ and $N(\mathcal{B})$ are effectively computed, one can construct winning strategies in Wadge games $W(L(\mathcal{A}), L(\mathcal{B}))$ and $W(L(\mathcal{B}), L(\mathcal{A}))$. These strategies may be defined by blind counter transducers, and this extends Wagner's result to blind counter automata.

\section{Concluding Remarks}

This extended abstract is still a very summarized presentation of our results, which will need exposition of many other details we could not include in this paper Fin00a.

We have considered above deterministic real time blind counter automata, which form a subclass of the class of deterministic pushdown automata and of the class of deterministic $k$-blind counter automata. The Wadge hierarchies of $\omega$ languages in each of these classes have been determined in a non effective way, by other methods, in Dup99b Fin99b Fin00b, and these results had been announced in the survey [DFR01]. The Wadge degrees in these hierarchies may be described with similar names

$$
N(\mathcal{A})=E_{m_{1}}^{\alpha_{1}} \ldots E_{m_{k}}^{\alpha_{k}} H_{m_{k+1}}^{\alpha_{k+1}}
$$

where $m_{1}>m_{2}>\ldots>m_{k}>m_{k+1}$ are integers $\geq 1$ and $H \in\{C, D, E\}$, and

1. each $\alpha_{i}$ is an ordinal $<\omega^{k+1}$, in the case of $k$-blind counter automata.

2. each $\alpha_{i}$ is an ordinal $<\omega^{\omega}$, in the case of deterministic pushdown automata.

We will further extend the results of the present paper in both directions to get decidability results and effective winning strategies in Wadge games. The above case of (one) blind counter automata already introduces some of the fundamental ideas which we will apply in further cases. 
Another problem is to study the complexity of the problem: " determine the Wadge degree of a blind counter $\omega$-language ", extending this way the results of Wilke and Yoo to blind counter $\omega$-languages.

Further study would be the investigation of links between the problems of simulation and bisimulation [Jan00, JKM00, JMS99, Kuc00 and the problem of finding winning strategies in Wadge games.

A Wadge game between two blind counter $\omega$-languages, whose complements are also blind counter $\omega$-languages, can easily be reduced to a Gale-stewart game, (see Tho95] PP98]), with a winning set accepted by a deterministic 2-blind-counter automaton. This suggests that Walukiewicz's result, the proof of the existence of effective winning strategies in a Gale-stewart game with a winning set accepted by a deterministic pushdown automaton, Wal96, could be extended to the case of a winning set accepted by a deterministic multi blind counter automata, giving additional results as asked by Thomas in Tho95.

Acknowledgements. Thanks to Jean-Pierre Ressayre and Jacques Duparc for many helpful discussions about Wadge and Wagner Hierarchies.

Thanks also to the anonymous referees for useful comments on the preliminary version of this paper. In particular the remark 19 is due to one of them.

\section{References}

BL69. J.R. Büchi and L. H. Landweber, Solving sequential conditions by finite state strategies. Trans. Amer. Math. Soc. 138 (1969).

CP97. O. Carton and D. Perrin, Chains and Superchains for $\omega$-Rational sets, Automata and semigroups, International Journal of Algebra and Computation Vol. 7, N. 7(1997) p. 673-695.

CP98. O. Carton and D. Perrin, The Wagner Hierarchy of $\omega$-Rational sets, International Journal of Algebra and Computation, vol. 9, no. 5, pp. 597-620, 1999.

Dup99a. J. Duparc, Wadge Hierarchy and Veblen hierarchy: part 1: Borel sets of finite rank, Journal of Symbolic Logic, March 2001.

Dup99b. J. Duparc, A Hierarchy of Context Free Omega Languages, Theoretical Computer Science, to appear. Available from http://www.logigue.jussieu.fr/www.duparc

DFR01. J. Duparc, O. Finkel and J-P. Ressayre, Computer Science and the Fine Structure of Borel Sets, Theoretical Computer Science, Volume 257 (1-2), April 2001, p.85-105.

EH93. J. Engelfriet and H. J. Hoogeboom, X-automata on $\omega$-words, Theoretical Computer Science 110 (1993) 1, 1-51.

Fin99a. O. Finkel, Wadge Hierarchy of Omega Context Free Languages, Theoretical Computer Science, to appear.

Fin99b. O. Finkel, Wadge Hierarchy of Deterministic Omega Context Free Languages, in preparation.

Fin00a. O. Finkel, An Effective Extension of the Wagner Hierarchy to Blind Counter Automata, full version, in preparation. 
Fin00b. O. Finkel, Wadge Hierarchy of Petri Net Omega Languages, in preparation. Gre78. S.A. Greibach, Remarks on Blind and Partially Blind One Way Multicounter Machines, Theoretical Computer Science 7 (1978) p. 311-324.

HU69. J.E. Hopcroft and J.D. Ullman, Formal Languages and their Relation to Automata, Addison-Wesley Publishing Company, Reading, Massachussetts, 1969.

Jan00. P. Jancar, Decidability of bisimilarity for one-counter processes, Information and Computation 158, 2000, pp. 1-17 (Academic Press) (A preliminary version appeared at ICALP'97.)

JKM00. P. Jancar, A. Kucera, and F. Moller, Simulation and Bisimulation over OneCounter Processes, In Proceedings of 17th International Symposium on Theoretical Aspects of Computer Science (STACS 2000), pages 334-345, volume 1770 of LNCS, Springer-Verlag, 2000.

JMS99. P. Jancar, F. Moller and Z. Sawa, Simulation Problems for One-Counter Machines, in Proc. SOFSEM'99 (Milovy, Czech Rep., November 1999), Lecture Notes in Computer Science, Vol. 1725, Springer 1999, pp. 404-413.

Kuc00. A. Kucera, Efficient Verification Algorithms for One-Counter Processes, In Proceedings of 27th International Colloquium on Automata, Languages, and Programming (ICALP 2000), pages 317-328, volume 1853 of LNCS, Springer-Verlag, 2000.

Kur66. K. Kuratowski, Topology, Academic Press, New York 1966.

Lan69. L. H. Landweber, Decision problems for $\omega$-automata, Math. Syst. Theory 3 (1969) 4,376-384.

Lin77. M. Linna, A decidability result for deterministic $\omega$-context-free languages, Theoretical Computer Science 4 (1977), 83-98.

LT94. H. Lescow and W. Thomas, Logical specifications of infinite computations, In:" A Decade of Concurrency" (J. W. de Bakker et al., eds), Springer LNCS 803 (1994), 583-621.

PP98. D. Perrin and J.-E. Pin, Infinite Words, Book in preparation, available from http://www.liafa.jussieu.fr/ jep/InfiniteWords.html

Sel98. V. Selivanov, Fine hierarchy of regular $\omega$-languages, Theoretical Computer Science 191(1998) p.37-59.

Sta86. L. Staiger, Hierarchies of Recursive $\omega$-Languages, Jour. Inform. Process. Cybernetics EIK 22 (1986) 5/6, 219-241.

Sta97a. L. Staiger, $\omega$-languages, Chapter of the Handbook of Formal Languages, Vol 3, edited by G. Rozenberg and A. Salomaa, Springer-Verlag, Berlin, 1997.

SW74. L. Staiger and K. Wagner, Automatentheoretische und Automatenfreie Charakterisierungen Topologischer Klassen Regulärer Folgenmengen. Elektron. Informationsverarb. Kybernetik EIK 10 (1974) 7, 379-392.

Tho90. W. Thomas, Automata on Infinite Objects, in: J. Van Leeuwen, ed., Handbook of Theorical Computer Science, Vol. B ( Elsevier, Amsterdam, 1990 ), p. 133-191.

Tho95. W. Thomas, On the synthesis of strategies in infinite games, in STACS'95, Volume 900 of LNCS, p.1-13, 1995.

Wad84. W.W. Wadge, Ph. D. Thesis, Berkeley, 1984.

Wag79. K. Wagner, On Omega Regular Sets, Inform. and Control 43 (1979) p. 123-177.

Wal96. I. Walukiewicz, Pushdown Processes: Games and Model Checking, Information and Computation 164 (2) p. 234-263, 2001.

WY95. Th. Wilke and H. Yoo, Computing the Wadge Degree, the Lifschitz Degree and the Rabin Index of a Regular Language of Infinite Words in Polynomial Time, in: TAPSOFT' 95: Theory and Practice of Software Development (eds. P.D. Mosses, M. Nielsen and M.I. Schwartzbach), L.N.C.S. 915, p. 288-302, 1995. 